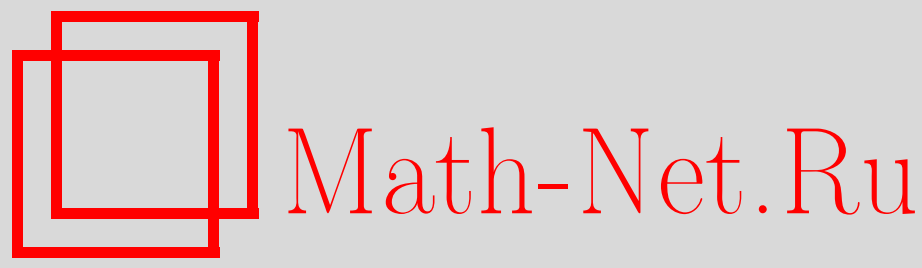

С. Ю. Садов, Об асимптотически свободном действии групп перестановок на подмножествах и мультимножествах, Дискрет. матем., 2014, том 26, выпуск 3, 101-120

DOI: https://doi.org/10.4213/dm1294

Использование Общероссийского математического портала Math-Net.Ru подразумевает, что вы прочитали и согласны с пользовательским соглашением http://www . mathnet.ru/rus/agreement

Параметры загрузки:

IP : 107.22 .136 .117

26 апреля 2023 г., 15:28:54

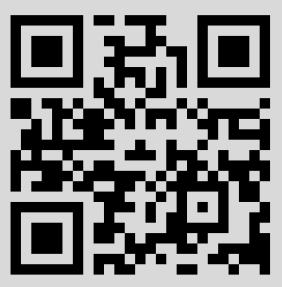




\title{
Об асимптотически свободном действии групп перестановок на подмножествах и мультимножествах
}

() 2014 г. С. Ю. Садов

\begin{abstract}
Пусть $G$ - группа перестановок, действующая на конечном множестве $\Omega$ мощности $n$. Для числа орбит индуцированного действия $G$ на множестве $\Omega_{m}$ всех подмножеств $\Omega$ мощности $m$ имеют место тривиальные оценки $\left|\Omega_{m}\right| /|G| \leqslant$ $\left|\Omega_{m} / G\right| \leqslant\left|\Omega_{m}\right|$. В статье даны улучшения верхней оценки в терминах минимальной степени группы $G$ или минимальной степени ее подмножества, дополнение которого мало. В частности, с использованием универсальных оценок Бохерта для минимальной степени группы и Бабаи-Пибера для порядка группы в терминах одного лишь $n$ показано, что если $G$ - произвольная 2 -транзитивная группа, за исключением симметрической и знакопеременной, $m$ и $n$ велики и отношение $m / n$ отделено от 0 и 1 , то $\left|\Omega_{m} / G\right| \approx\left|\Omega_{m}\right| /|G|$.

Аналогичные результаты верны для индуцированного действия $G$ на множестве $\Omega_{(m)}$ всех мультимножеств $\Omega$ веса $m$, если отношение $m /(m+n)$ равномерно отделено от 0 и 1.
\end{abstract}

Ключевые слова: группа перестановок, регулярные орбиты, средний размер стабилизатора, минимальная степень группы, асимптотика числа орбит, перечисление аффинных конфигураций, перечисление графов, асимптотически свободное действие.

\section{1. Введение}

Пусть $\Omega$ - конечное множество мощности $|\Omega|=n, G$ - группа перестановок, действующая на $\Omega$, т.е. подгруппа полной симметрической группы $\operatorname{Sym}(\Omega)$. Обозначим через $\Omega_{m}$ множество всех $m$-элементных подмножеств в $\Omega$, а через $\Omega_{(m)}-$ множество всех $m$-элементных мультимножеств на $\Omega$, т.е. функций кратности $\varkappa: \Omega \rightarrow \mathbb{Z}_{+}$таких, что $\sum_{\omega \in \Omega} \varkappa(\omega)=m$; здесь $\mathbb{Z}_{+}-$множество неотрицательных целых чисел. Группа $G$ естественным образом действует на $\Omega_{m}$ и $\Omega_{(m)}$. Нас интересует оценка числа орбит этих действий при больших $n$ и $m$. Неформально говоря, основной тезис работы состоит в том, что во многих естественных ситуациях средний размер стабилизатора множества $x \in \Omega_{m}$ или мультимножества $x \in \Omega_{(m)}$ близок к единице, стабилизатор $G_{x}$ для большинства таких $x$ состоит из тождественного

* Место работы: Институт прикладной математики им. М. В. Келдыша РАН, e-mail: serge.sadov@gmail.com 
преобразования, а орбиты $G x$ регулярны, т.е. имеют максимальный возможный размер, $|G|$. Соответственно, числа орбит $n_{m}(G)=\left|\Omega_{m} / G\right|$ и $n_{(m)}(G)=\left|\Omega_{(m)} / G\right|$ близки к минимально возможным, соответствующим свободному действию, а именно, $n_{m}(G) \sim n_{m} /|G|$ и $n_{(m)}(G) \sim n_{(m)} /|G|$, где $n_{m}=\left|\Omega_{m}\right|, n_{(m)}=\left|\Omega_{(m)}\right|$.

Асимптотическая свобода действия группы перестановок на (мульти)множествах - явление, давно подмеченное в отдельных содержательных задачах перечислительной комбинаторики. Наиболее ранний известный мне из литературы пример - результат об асимптотике по числу вершин количества графов (с точностью до изоморфизма). ${ }^{1}$ В нашем контексте проще обсуждать число $g_{v, m}$ графов с заданными числами вершин $|V|=v$ и ребер $m$. Тогда $\Omega=V_{2}-$ множество пар вершин, $n=|\Omega|=\left(\begin{array}{l}v \\ 2\end{array}\right)$; элементы $\Omega_{m}$ соответствуют помеченным графам с $m$ ребрами, $G=\operatorname{Sym}(V)$ - группа перенумераций вершин графа, факторизация $\Omega_{m}$ по действию $G$ соответствует отождествлению графов, отличающихся лишь порядком меток, так что $g_{v, m}=\left|\Omega_{m} / G\right|$. Если отношение $m /|\Omega|-$ плотность единиц в матрице инцидентности графа - не слишком близко к 0 и 1 , то $g_{v, m} \sim\left|\Omega_{m}\right| /|G|$, т.е. действие почти свободно [3], [1; упр. 9.13]. В этом примере и вообще нередко задача перечисления помеченных структур несложна, и главный вопрос заключается в количественном учете отождествлений вследствие «устранения меток».

Богатый материал для изучения вопроса о существовании и типичности регулярных орбит дает линейная алгебра над конечными полями: действия линейных (аффиных, проективных) групп на конфигурациях точек, грассманианах и т.д. В [4] доказано существование подмножеств линейного пространства $V$ размерности не меньше 5 над $\mathbb{F}_{2}$ с единичным стабилизатором ${ }^{2}$ относительно действия полной линейной группы, т.е. установлен факт существования регулярных орбит $G L(V)$ в $2^{V}$. В работе [5], с. 166, говоря о классах эквивалентности булевых функций относительно линейных групп, автор замечает (основываясь на фольклоре или интуиции?) что «асимптотически почти все функции и типы имеют единичный стабилизатор», не приводя точных формулировок и ссылок.

К линейной алгебре относятся результаты Уальда $[6,7]$ и Хоу $[8,9,10]$ об асимптотическом перечислении матроидов и $q$-ичных кодов; показано, что действие группы перестановок элементов фиксированного базиса на грассманиане в $\mathbb{F}_{q}^{n}$ асимптотически $(n \rightarrow \infty)$ свободно. В статье [11] на ту же тему затрагивается привлекательная и не отраженная в настоящей работе точка зрения - связь с асимптотической теорией характеров.

Большое число работ - см., например, $[12,13,14]$ - посвящено другим аспектам действия $G$ на множествах подмножеств $\Omega_{m}$, включая и случай, когда множество $\Omega$ бесконечно $(n=\infty)$, но количества орбит $n_{m}(G)$ конечны. В [15] доказано интуитивно ожидаемое свойство: последовательность $n_{k}(G)$ унимодальна, т.е. меняет направление монотонности лишь однажды; более точно, она не убывает при $m \leqslant n / 2$. (А при $m>n / 2$ имеем $n_{m}(G)=n_{n-m}(G)$.) Однако вопросом об асимптотике чисел $n_{m}(G)$ при больших, но конечных значениях одновременно $n$ и $m$ авторы работ

\footnotetext{
${ }^{1}$ По свидетельству Ф. Харари [1; гл. 9], об этом результате ему сообщил Д. Пойа в письме в начале 1950-х гг. Процитирую ссылку (2) из статьи [2]: «R.J. Riddell, "Contributions to the Theory of Condensation" (dissertation, University of Michigan, 1951); R.J. Riddell and G.E. Uhlenbeck, "On the Theory of the Virial Development of the Equation of State of Monoatomic Gases," J. Chem. Phys., 21, 2056, 1953. The contributions of Polya are given in these references but have not been published by Polya himself.»

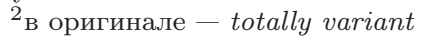


указанного направления, видимо, не задавались - иначе оценки в духе излагаемых ниже были бы, несомненно, упоминаемы.

Настоящая работа возникла как ответвление в проекте по перечислению тонких градуировок на алгебрах Ли. Я благодарен М.В. Кочетову за предложение принять участие в этом проекте. Задача о нетривиальной оценке среднего размера стабилизатора возникла при нашем совместном обсуждении возможного улучшения верхней оценки в Теореме 6.2 работы [16] (будет опубликовано отдельно).

О структуре работы. Работа состоит из «теоретической части» (\$§ 2-4) и приложений (§ 5). Итоговые результаты теоретической части - теоремы 4 и 5 об оценках среднего размера стабилизатора действия $G$ на $\Omega_{m}$ и $\Omega_{(m)}$. О связи этих величин с числами орбит $n_{m}, n_{(m)}$ см. начало $\S 4$. Подсчет числа орбит базируется на формуле Бернсайда, поэтому наш анализ начинается с оценки числа подмножеств или мультимножеств, инвариантных относительно одной перестановки, в терминах ее цикловой структуры. Этому посвящен $\S 2$ : в нем сосредоточена вся «аналитическая» часть. Наши неравенства элементарны, но заведомо не оптимальны: приоритет отдан простоте и универсальности оценок.

В $\S 3$ и 4 последовательно расширяется круг задействованных объектов; результаты переносятся сначала на произвольные совокупности подстановок (§ 3$)$, а потом на группы (§ 4). Доказательства новых утверждений в этих параграфах сводятся к тривиальным подстановкам. Большинство используемых понятий стандартно, но укажу два исключения. (1) В $\$ 3$ определяется цикловой индекс для произвольной совокупности перестановок; в случае групп он отличается от циклового индекса теории перечисления Редфилда-Пойа отсутствием нормализующего множителя. (2) Часто встречающийся объект - пара $(\sigma, x)$, где $x$ - неподвижная точка перестановки $\sigma$, - кажется, не имеет общепринятого названия. Я использую термин «пассивная пара».

Теоремы 1, 2, 3 и 4 составляют логическую цепочку, в которой происходят последовательные огрубления оценок и, таким образом, потенциально присутствует возможность более полного использования доступной информации о цикловой структуре отдельных перестановок и их совокупностей. Вариацию на эту тему представляет ответвление «Теорема $3 \Rightarrow$ Следствие $1 \Rightarrow$ Теорема 5 ».

Предложение 1 , завершающее $\S 4$, - это строгая формулировка утверждения о том, что если средний размер стабилизатора близок к единице, то почти все орбиты регулярны.

Из трех разделов $\S 5$ в двух описаны примеры применения теорем 4 и 5 к конкретным группам, а в разделе 5.3 обсуждаются следствия теоремы 4 в свете результатов общей теории групп перестановок, восходящих к работам XIX века Жордана и Бохерта.

Теорема 6 в разделе 5.1 о действии $G L(d, q)$ на подмножествах/ мультимножествах - иллюстрация эффективности теоремы 4 в простой ситуации, которая, однако, может представлять достаточно общий интерес. Мне неизвестны публикации, содержащие этот результат. Запланированное приложение теоремы 6 - улучшение оценки из [16], о котором сказано выше.

K сожалению, ни теорема 6 , ни - напрямую - теорема 4 неприменимы для вывода упомянутых ранее результатов Уальда и Хоу об асимптотике числа матроидов или линейных кодов. Было бы, тем не менее, интересно вывести эти результаты в рамках объединяющего подхода. По-видимому, требуется уточнение доказательства теоремы 1 с использованием, хотя бы частично, той дополнительной информации, 
которая сработала у Уальда и Хоу (теории нормальных форм матриц над конечным полем).

Цель раздела 5.2 - на примере проиллюстрировать сходство и различие применения теорем 4 и 5 (к асимптотическому перечислению, соответственно, однородных гиперграфов и графов). Теорема 8 перекрывает теорему Обершельпа [1; § 9.3], но слабее теоремы Райта [3].

Главная цель раздела 5.3 - посмотреть, что дают при подстановке в теорему 4 известные неравенства, связывающие степень группы перестановок с ее порядком и минимальной степенью. Неожиданно общий вывод (теорема 9b) получается для 2-транзитивных групп, за исключением полной симметрической и знакопеременной: такие группы действуют почти свободно при $n=|\Omega| \rightarrow \infty$ на $\Omega_{m}$, если отношение $m / n$ отделено от 0 и 1 , и на $\Omega_{(m)}$, если $m /(m+n)$ отделено от 0 и 1.

\section{2. Оценки для одной перестановки}

Сначала оценим число множеств и мультимножеств данного размера, инвариантных относительно данной перестановки, в терминах длин циклов этой перестановки.

1. Напомним обозначения: $n=|\Omega|, n_{m}=\left|\Omega_{m}\right|$ (количество всех $m$-элементных подмножеств множества $\Omega, 0 \leqslant m \leqslant n$ ), и $n_{(m)}=\left|\Omega_{(m)}\right|$ (количество всех $m$-мультимножеств в $\Omega, m \geqslant 0)$. Имеем

$$
n_{m}=\left(\begin{array}{c}
n \\
m
\end{array}\right), \quad n_{(m)}=\left(\begin{array}{c}
n+m-1 \\
m
\end{array}\right) .
$$

Зафиксируем обозначения для отношений $m / n$ и $m /(n+m)$ :

$$
\frac{m}{n}=\lambda, \quad \frac{m}{n+m}=\eta .
$$

Вводя обозначение

$$
h(p)=-p \ln p-q \ln q
$$

для энтропии двузначного распределения с вероятностями $p$ и $q=1-p$, по формуле Стирлинга имеем

$$
n_{m}=\frac{e^{n h(\lambda)}}{\sqrt{2 \pi n \lambda(1-\lambda)}}\left(1+O\left(n^{-1}\right)\right)
$$

И

$$
n_{(m)}=\frac{e^{(n+m) h(\eta)}}{\sqrt{2 \pi(n+m)}} \sqrt{\frac{1-\eta}{\eta}}\left(1+O\left(n^{-1}\right)\right) .
$$

Постоянные в $O$-оценках равномерно ограничены, когда $\lambda$ и $\eta$ отделены от 0 и 1 . (Более точно, предположение $\lambda \in[\varepsilon, 1-\varepsilon]$ относится к формуле (3), а предположение $\eta \in[\varepsilon, 1-\varepsilon]-$ к формуле (4).)

2. Для перестановки $\sigma \in \operatorname{Sym}(\Omega)$, пусть $j_{k}(\sigma)$ - число циклов длины $k$, так что $\sum_{k=1}^{n} k j_{k}=n$. Удобный вспомогательный объект - цикловой моном

$$
z_{\sigma}\left(t_{1}, \ldots, t_{n}\right)=\prod_{k \geqslant 1} t_{k}^{j_{k}(\sigma)} .
$$

Здесь $t_{1}, t_{2}, \ldots$ - формальные переменные. Ясно, что $z_{\sigma}\left(t, t^{2}, t^{3}, \ldots\right)=t^{n}$. Положим

$$
\alpha(\sigma)=j_{1}(\sigma)
$$


И

$$
\beta(\sigma)=n-\alpha(\sigma)=\sum_{k \geqslant 2} k j_{k}(\sigma) .
$$

Таким образом, $\alpha(\sigma)=|\operatorname{Fix}(\sigma)|$ - число неподвижных точек перестановки $\sigma$, а число $\beta(\sigma)$, называемое степенъю перестановки $\sigma$, см. [17], - размер носителя $\operatorname{supp}(\sigma)=$ $\Omega \backslash \operatorname{Fix}(\sigma)=\{\omega \in \Omega \mid \sigma(\omega) \neq \omega\}$.

Введем обозначения для чисел неподвижных точек действия $\sigma$ на $\Omega_{m}$ и $\Omega_{(m)}$ :

$$
\alpha_{m}(\sigma)=\left|\operatorname{Fix}_{m}(\sigma)\right|, \quad \alpha_{(m)}(\sigma)=\left|\operatorname{Fix}_{(m)}(\sigma)\right|,
$$

где $\operatorname{Fix}_{m}(\sigma) \subset 2^{\Omega_{m}}-$ множество $\sigma$-инвариантных $m$-подмножеств в $\Omega, \operatorname{a~Fix}_{(m)}(\sigma) \subset$ $2^{\Omega_{(m)}}-$ множество $\sigma$-инвариантных $m$-мультимножеств.

Производящими функциями для величин (8) будут

$$
A_{\sigma}(t)=\sum_{m=0}^{n} \alpha_{m}(\sigma) t^{m}
$$

И

$$
A_{(\sigma)}(t)=\sum_{m=0}^{\infty} \alpha_{(m)}(\sigma) t^{m} .
$$

Хорошо известно и элементарно выводится тождество

$$
A_{\sigma}(t)=\prod_{k \geqslant 1}\left(1+t^{k}\right)^{j_{k}(\sigma)}=z_{\sigma}\left(1+t, 1+t^{2}, 1+t^{3}, \ldots\right) .
$$

В самом деле, $\Omega$ есть объединение $\sigma$-орбит (циклов перестановки $\sigma$ ). Всякое $\sigma$-инвариантное подмножество $x \subset \Omega$ однозначно соответствует подмножеству $\bar{x}$ пространства орбит $\Omega / \sigma$. При раскрытии скобок в произведении $P=\prod_{\xi \in \Omega / \sigma}\left(1+\tau_{\xi}\right)$ подмножеству $\bar{x}$ соответствует единственный моном $p_{x}=\prod_{\xi \in \bar{x}} \tau_{\xi}$. Подставляя $\tau_{\xi}=t^{|\xi|}$, получим $P=\prod_{k \geqslant 1}\left(1+t^{k}\right)^{j_{k}(\sigma)}$, а моном $p_{x}$ обращается в $t^{k}$ с показателем $k=\sum_{\xi \in \bar{x}}|\xi|=|x|$, равным размеру подмножества $x$.

Аналогично имеет место тождество

$$
A_{(\sigma)}(t)=\prod_{k \geqslant 1}\left(1-t^{k}\right)^{-j_{k}(\sigma)}=z_{\sigma}\left(\frac{1}{1-t}, \frac{1}{1-t^{2}}, \ldots\right) .
$$

В частности, когда $\sigma=e$ (тождественная подстановка), имеем

$$
A_{e}(t)=(1+t)^{n}, \quad A_{(e)}(t)=(1-t)^{-n} .
$$

3. Отправной точкой для последующего анализа являются оценки числа $\sigma$-инвариантных $m$-(мульти)множеств. Напомним, что используемые в теореме 1 обозначения введены формулами (1), (2) и (6), (7), (8).

Теорема 1. Для перестановки б справедливь следующие верхние оценки числа б-инвариантных т-подмножеств и т-мультимножеств:

$$
\alpha_{m}(\sigma) \leqslant(1-2 \lambda(1-\lambda))^{\beta(\sigma) / 2} e^{n h(\lambda)}
$$

$u$

$$
\alpha_{(m)}(\sigma) \leqslant\left(\frac{1-\eta}{1+\eta}\right)^{\beta(\sigma) / 2} e^{(n+m) h(\eta)} .
$$


Доказательство. (а) При любом $\tau>0$ функция $k \mapsto\left(1+\tau^{k}\right)^{1 / k}$ убывает. Следовательно, справедливо неравенство

$$
1+\tau^{k} \leqslant\left(1+\tau^{2}\right)^{k / 2}, \quad k \geqslant 2 .
$$

Применяя это неравенство с $\tau=t^{3}, t^{4}, \ldots$, находим

$$
A_{\sigma}(t) \leqslant(1+t)^{\alpha(\sigma)}\left(1+t^{2}\right)^{\beta(\sigma) / 2}=A_{e}(t)\left(\frac{1+t^{2}}{(1+t)^{2}}\right)^{\beta(\sigma) / 2} .
$$

Поскольку коэффициенты производящей функции $A_{\sigma}(t)$ неотрицательны, имеем: для любого $t>0$

$$
\alpha_{m}(\sigma) \leqslant t^{-m} A_{\sigma}(t) \leqslant t^{-m} A_{e}(t)\left(\frac{1+t^{2}}{(1+t)^{2}}\right)^{\beta(\sigma) / 2} .
$$

Минимум функции $t^{-m} A_{e}(t)=t^{-m}(1+t)^{n}$ достигается в точке

$$
t_{*}=\frac{m}{n-m}=\frac{\lambda}{1-\lambda} .
$$

Вычисляя, находим

$$
\frac{1}{1+t_{*}}=1-\lambda, \quad \frac{t_{*}}{1+t_{*}}=\lambda
$$

и

$$
t_{*}^{-m} A_{e}\left(t_{*}\right)=\left[\lambda^{\lambda}(1-\lambda)^{1-\lambda}\right]^{-n}=e^{n h(\lambda)} .
$$

Имеем также

$$
\frac{1+t_{*}^{2}}{\left(1+t_{*}\right)^{2}}=1-\frac{2 t_{*}}{\left(1+t_{*}\right)^{2}}=1-2 \lambda(1-\lambda) .
$$

Используя значение $t=t_{*}$ в оценке (12), придем к неравенству (9).

(b) Доказательство неравенства (10) полностью аналогично. Вместо (11) используем неравенство

$$
\left(1-\tau^{k}\right)^{-1} \leqslant\left(1-\tau^{2}\right)^{-k / 2}, \quad k \geqslant 2,
$$

справедливое при любом $\tau \in[0,1)$.

Подставляя $\tau=t^{3}, t^{4}, \ldots$, находим

$$
A_{(\sigma)}(t) \leqslant(1-t)^{\alpha(\sigma)}\left(1-t^{2}\right)^{-\beta(\sigma) / 2}=A_{(e)}(t)\left(\frac{1-t}{1+t}\right)^{\beta(\sigma) / 2} .
$$

Как и в (12), имеем: при любом $t \in(0,1)$

$$
\alpha_{(m)}(\sigma) \leqslant t^{-m} A_{(\sigma)}(t) \leqslant t^{-m} A_{(e)}(t)\left(\frac{1-t}{1+t}\right)^{\beta(\sigma) / 2} .
$$

В правой части в качестве $t$ используем значение

$$
t_{(*)}=\frac{m}{n+m}=\eta,
$$

доставляющее минимум функции $t^{-m} A_{(e)}(t)=t^{-m}(1-t)^{-n}$. Значение минимума есть $t_{(*)}^{-m} A_{(e)}\left(t_{(*)}\right)=e^{(m+n) h(\eta)}$, и теперь из $(13)$ следует $(10)$. 


\section{3. Оценки для совокупности перестановок}

Назовем пару $(\sigma, x)$, где $x$ - мультимножество (в частности, подмножество) на $\Omega$, nассивной nарой, если $\sigma(x)=x$. Наша цель в этом разделе - получить общие оценки для числа пассивных пар $(\sigma, x)$ с определенными ограничениями на $\sigma$ и $x$.

Пусть $K-$ подмножество (не обязательно подгруппа) группы $\operatorname{Sym}(\Omega)$. Вся информация о цикловой структуре перестановок совокупности $K$ содержится в цикловом индексе - сумме цикловых мономов вида (5)

$$
z^{K}\left(t_{1}, \ldots, t_{n}\right)=\sum_{\sigma \in K} z_{\sigma}\left(t_{1}, \ldots, t_{n}\right) .
$$

Нам понадобится производящая функция более частного вида, содержащая лишь информацию о распределении числа неподвижных точек или, эквивалентно, о распределении размеров носителей. Пусть $f_{k}^{K}-$ количество таких $\sigma \in K$, для которых $\beta(\sigma)=k$. Положим

$$
F^{K}(t)=\sum_{k=0}^{n} f_{k}^{K} t^{k}
$$

Легко видеть, что

$$
F^{K}(t)=\sum_{\sigma \in K} t^{\beta(\sigma)}=z^{K}\left(1, t^{2}, t^{3}, \ldots\right)
$$

Пусть $u_{m}^{K}$ (соответственно, $\left.u_{(m)}^{K}\right)$ - число таких пассивных пар $(\sigma, x)$, для которых $\sigma \in K$ и $x \in \Omega_{m}$ (соответственно, $\left.\sigma \in K, x \in \Omega_{(m)}\right)$. Очевидны равенства

$$
u_{m}^{K}=\sum_{\sigma \in K} \alpha_{m}(\sigma), \quad u_{(m)}^{K}=\sum_{\sigma \in K} \alpha_{(m)}(\sigma) .
$$

Удобно ввести обозначения

$$
\begin{aligned}
& \xi_{1}=\xi_{1}(\lambda)=(1-2 \lambda(1-\lambda))^{1 / 2}=\frac{\sqrt{m^{2}+(n-m)^{2}}}{n} \\
& \xi_{2}=\xi_{2}(\eta)=\left(\frac{1-\eta}{1+\eta}\right)^{1 / 2}=\left(\frac{n}{n+2 m}\right)^{1 / 2} .
\end{aligned}
$$

Сумма по $\sigma \in K$ оценок из теоремы 1 дает следующий результат.

Теорема 2. Имеют место неравенства

$$
u_{m}^{K} \leqslant e^{n h(\lambda)} F^{K}\left(\xi_{1}(\lambda)\right)
$$

$u$

$$
u_{(m)}^{K} \leqslant e^{(n+m) h(\eta)} F^{K}\left(\xi_{2}(\eta)\right) .
$$

Определение. Назовем минимальной степенъю подмножества $K \subset \operatorname{Sym}(\Omega)$ минимальный размер носителя перестановки $\sigma \in K$ :

$$
\mu^{K}=\min _{\sigma \in K} \beta(\sigma)
$$

Замечание. Если $e \in K$, наше определение дает $\mu^{K}=0$. В частности, так обстоит дело, когда $K$ - группа. В этом случае для разрешения терминологической 
коллизии приходится разделять понятия минимальной степени подмножества и минимальной степени группы. Согласно стандартному определению (см., например, [17]), минимальная степень группы $G$ равна минимальной степени подмножества $G^{\prime}=G \backslash\{e\}$ в нашем смысле. В явном виде,

$$
\mu^{G^{\prime}}=\min _{g \in G, g \neq e}|\{\omega \in \Omega \mid g(\omega) \neq \omega\}| .
$$

Теорема 3. Числа пассивных пар очениваются сверху через минимальную степень $\mu=\mu^{K}$ подмножества $K$ следующим образом:

$$
u_{m}^{K} \leqslant|K| e^{n h(\lambda)} \xi_{1}(\lambda)^{\mu}
$$

$u$

$$
u_{(m)}^{K} \leqslant|K| e^{(n+m) h(\eta)} \xi_{2}(\eta)^{\mu} .
$$

Доказательство. Используем теорему 2 и оценку $F^{K}(\xi) \leqslant|K| \xi^{\mu}$.

Величины $u_{m}^{K}$ и $u_{(m)}^{K}$, рассматриваемые как функции множества $K$, аддитивны. Это очевидное замечание может быть полезно для изоляции влияния «плохого» множества $B$ малого размера на оценку в целом:

Следствие 1. Пусть $B \subsetneq K u \mu^{*}:=\mu^{K \backslash B}>\mu$. Тогда оценки (17) $и$ (18) можно улучшить:

$$
u_{m}^{K} \leqslant e^{n h(\lambda)}\left((|K|-|B|) \xi_{1}(\lambda)^{\mu^{*}}+|B| \xi_{1}(\lambda)^{\mu}\right)
$$

$u$

$$
u_{(m)}^{K} \leqslant e^{(n+m) h(\eta)}\left((|K|-|B|) \xi_{2}(\eta)^{\mu^{*}}+|B| \xi_{2}(\eta)^{\mu}\right) .
$$

\section{4. Оценки среднего размера стабилизатора}

Обратимся к выводу оценок, относящихся к действию группы $G \subset \operatorname{Sym}(\Omega)$ на множестве подмножеств $\Omega_{m}$ и мультимножеств $\Omega_{(m)}$.

Вообще, если $S$ - конечное множество, на котором действует $G$, то, как известно, число орбит $|S / G|$ дается формулой Бернсайда (или Коши-Фробениуса-Бернсайда)

$$
|S / G|=\frac{1}{|G|} \sum_{\sigma \in G}\left|\operatorname{Fix}_{S}(\sigma)\right|, \quad \operatorname{Fix}_{S}(\sigma)=\{s \in S \mid \sigma(s)=s\} .
$$

В этом виде формула часто использутся в вычислениях, но для формулировки и интерпретации наших результатов будет удобнее ее альтернативная форма

$$
|S / G|=\frac{|S|}{|G|}\left\langle\operatorname{Stab}_{S}^{G}\right\rangle
$$

где

$$
\left\langle\operatorname{Stab}_{S}^{G}\right\rangle=\frac{1}{|S|} \sum_{s \in S}|\operatorname{Stab}(s)|
$$

- средний размер стабилизатора элемента $s \in S$.

Тривиальным образом $1 \leqslant\left\langle\operatorname{Stab}_{S}^{G}\right\rangle \leqslant|G| ;$ соответственно,

$$
\frac{|S|}{|G|} \leqslant|S / G| \leqslant|S|
$$


Верхняя граница отвечает случаю тривиального действия, когда все орбиты одноточечны. Нижняя граница отвечает случаю свободного действия, когда все нетождественные перестановки $\sigma \in G$ действуют без неподвижных точек.

В случае $|S| \leqslant|G|$ очевидна нижняя оценка $|S / G| \geqslant 1$ (равенство - если действие транзитивно); соответственно, $\left\langle\operatorname{Stab}_{S}^{G}\right\rangle \geqslant|G| /|S|$.

Объектом нашего внимания будут улучшения верхней оценки в (21) и условия, при которых улучшенная оценка асимптотически сближается с тривиальной нижней. В качестве множества $S$ будут выступать $\Omega_{m}$ и $\Omega_{(m)}$.

Считая группу $G$ и множество $\Omega$ фиксированными, будем писать $\left\langle\operatorname{Stab}_{m}\right\rangle$ вместо $\left\langle\operatorname{Stab}_{\Omega_{m}}^{G}\right\rangle$ и $\left\langle\operatorname{Stab}_{(m)}\right\rangle$ вместо $\left\langle\operatorname{Stab}_{\Omega_{(m)}}^{G}\right\rangle$.

С учетом введенных ранее обозначений $\left(n_{m}=\left|\Omega_{m}\right|, u_{m}^{G}\right.$ - число пассивных пар в $G \times \Omega_{m}$ и т.п.), средний размер стабилизатора в двух интересующих нас случаях дается формулами

$$
\left\langle\operatorname{Stab}_{m}\right\rangle=\frac{u_{m}^{G}}{n_{m}}, \quad\left\langle\operatorname{Stab}_{(m)}\right\rangle=\frac{u_{(m)}^{G}}{n_{(m)}} .
$$

Следующая теорема непосредственно следует из теоремы 3 и асимптотических формул (3), (4).

Теорема 4. Для любого $\varepsilon \in(0,1 / 2)$ nри всех достаточно больших $n>n_{0}(\varepsilon)$ верно следующее утверждение.

Пусть $\mu=\mu^{G^{\prime}}$ - минимальная степень группь перестановок $G$, действующей на множестве $\Omega$ размера $n$. Отклонение от 1 среднего размера стабилизатора естественного действия группы $G$ на множествах и мультимножествах размера $m$ в $\Omega$ допускает ощенки сверху:

$$
\frac{\left\langle\operatorname{Stab}_{m}\right\rangle-1}{|G|} \leqslant\left(C_{1}+\varepsilon\right) \sqrt{n} \cdot \xi_{1}^{\mu} \quad \text { npu } \lambda \in[\varepsilon, 1-\varepsilon]
$$

для действия на множествах и

$$
\frac{\left\langle\operatorname{Stab}_{(m)}\right\rangle-1}{|G|} \leqslant\left(C_{2}+\varepsilon\right) \sqrt{n+m} \cdot \xi_{2}^{\mu} \quad \text { npu } \eta \in[\varepsilon, 1-\varepsilon]
$$

для действия на мультимножествах. В этих оченках $\lambda=m / n, \eta=m /(m+n)$,

$$
C_{1}=\sqrt{2 \pi \lambda(1-\lambda)}, \quad C_{2}=\sqrt{2 \pi \eta /(1-\eta)},
$$

$a \xi_{1}=\xi_{1}(\lambda)$ u $\xi_{2}=\xi_{2}(\eta)-$ такие же, как в (14).

Насколько информативна теорема 4? Неравенства (22) и (23) содержательны, если их правые части меньше 1. Следовательно, «порог применимости» теоремы может быть охарактеризован соотношением

$$
\mu \approx \log n \text {. }
$$

Эффективность теоремы тем выше, чем больше отношение $\mu / \ln n$.

Отметим, что множители порядка $\sqrt{n}$ в правых частях оценок теоремы 4 имеют искусственное происхождение. Их присутствие - результат простого, грубого способа оценки коэффициентов производящих функций $A_{\sigma}(t)$ и $A_{(\sigma)}(t)$ в Теореме 1. 
Более аккуратные оценки с помощью метода перевала позволили бы избавиться от этих множителей и, таким образом, получить нетривиальные верхние оценки числа

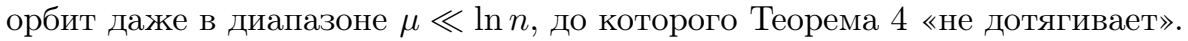

Итак, множители порядка $\sqrt{n}$ несущественны, когда $\mu \gg \ln n$. В силу известных результатов теории групп перестановок, цитируемых в $\S 5.3$, это условие выполнено (асимптотически) по крайней мере для транзитивных групп, кроме $\operatorname{Sym}(\Omega)$ и $A l t(\Omega)$. Более важен в теореме 4 вопрос о сравнительной величине $\mu$ и $\ln |G|$. Грубо говоря, теорема показывает, что действие $G$ на множествах и мультимножествах подходящего размера почти свободно при условии $\mu \gg \ln |G|$. Это условие можно ослаблять за счет использования более детальной информации о циклах перестановок группы $G$. Например, $\mu$ (минимальная степень группы) - не вполне удачный для наших целей параметр в том случае, если лишь малое число элементов группы (скажем, элементы «плохого» подмножества $B$ ) имеют близкую к $\mu$ или, во всяком случае, недостаточно хорошо оцениваемую снизу степень, в то время как степень большинства элементов намного превышает $\mu$, то есть $\mu^{G \backslash B} \gg \mu$.

Имея в виду ситуации подобного рода, приведем теорему, которая относится к теореме 4 так же, как к теореме 3 относится следствие 1.

Теорема 5. Пусть, в дополнение $\kappa$ условиям теоремы 4, для подмножества $B$ группы $G$ известно, что $\mu^{*}:=\mu^{G \backslash B}>\mu$. Тогда имеют место оценки

$$
\left\langle\operatorname{Stab}_{m}\right\rangle-1 \leqslant\left(C_{1}+\varepsilon\right) \sqrt{n}\left(|G| \xi_{1}^{\mu^{*}}+|B| \xi_{1}^{\mu}\right)
$$

$u$

$$
\left\langle\operatorname{Stab}_{(m)}\right\rangle-1 \leqslant\left(C_{2}+\varepsilon\right) \sqrt{n+m}\left(|G| \xi_{2}^{\mu^{*}}+|B| \xi_{2}^{\mu}\right) .
$$

с теми же обозначениями, что и в теореме 4.

Успех применения теоремы 5 для доказательства асимптотической свободы действия $G$ на (мульти)множествах зависит от возможности выделить такое «плохое, но малое» подмножество $B \subset G$, что

$$
\mu^{*} \gg \log |G|, \quad \mu \gg \log |B| .
$$

В заключение части статьи, посвященной общей теории, приведем не зависящие от нашей теории, но полезные для ее приложений нижние оценки числа регулярныx орбит и принадлежащих им точек. Их удобно представить в вероятностных терминах.

Предложение 1. Пусть группа $G$ действует на множестве $S$ и $\delta$-отклонение среднего значения стабилизатора от 1:

$$
\delta=\left\langle\operatorname{Stab}_{S}^{G}\right\rangle-1
$$

(а) Пусть на пространстве орбит $S / G$ задано равномерное вероятностное распределение (т.е. все орбиты равновероятны). Тогда вероятность того, что случайно выбранная орбита регулярна, не менъше $(1-\delta) /(1+\delta)$.

(b) Пусть на множестве $S$ задано равномерное вероятностное распределение (т.е. все точки равновероятны). Тогда вероятность того, что случайно выбранная точка s принадлежит регулярной орбите (эквивалентно - что $|\operatorname{Stab}(s)|=1)$, не менъwe $1-\delta$. 
Доказательство. (а) Пусть $R$ - множество регулярных орбит. Тогда

$$
\begin{array}{ccc}
\mathcal{O} \in R & \Rightarrow|\mathcal{O}|=|G|, \\
\mathcal{O} \notin R & \Rightarrow \quad|\mathcal{O}| \leqslant|G| / 2 .
\end{array}
$$

Следовательно,

$$
|S|=\sum_{\mathcal{O} \in S / G}|\mathcal{O}|=\sum_{\mathcal{O} \in R}+\sum_{\mathcal{O} \notin R} \leqslant|R||G|+(|S / G|-|R|) \frac{|G|}{2} .
$$

Деля обе части на $|G|$ и пользуясь равенством $|S| /|G|=|S / G|(1+\delta)^{-1}$, приходим к неравенству

$$
|S / G|\left(\frac{1}{1+\delta}-\frac{1}{2}\right) \leqslant \frac{|R|}{2},
$$

поэтому доля регулярных орбит, равная $|R| /|S / G|$, не меньше $(1-\delta) /(1+\delta)$.

(b) Возвращаясь к (26) и делая замену в правой части: $|S / G|=(1+\delta)|S| /|G|$, находим

$$
|R||G| \geqslant|S|(1-\delta) .
$$

Значит, что доля точек, принадлежащих регулярным орбитам, не меньше $1-\delta$.

\section{5. Приложения}

5.1. Число классов аффинной эквивалентности наборов векторов Возьмем в качестве $\Omega$ линейное пространство $V$ размерности $d$ над конечным полем $\mathbb{F}_{q}$. Перечисление классов аффинной эквивалентности $m$-точечных непомеченных конфигураций в пространстве $V$ равносильно перечислению орбит действия аффинной группы $G=A G L(d, q)$ на множестве $\Omega_{m}$ (если все точки конфигурации различны), либо на $\Omega_{(m)}$ (если допускаются кратные точки). Следуя обозначениям в начале статьи, назовем эти числа орбит соответственно $n_{m}(A G L(d, q))$ и $n_{(m)}(A G L(d, q))$.

Пусть $q$ фиксировано, а $d, m \rightarrow \infty$. Отметим, что

$$
\begin{gathered}
n=|\Omega|=q^{d} \\
|G|=|G L(d, q)|+|V|=\prod_{j=0}^{d-1}\left(q^{d}-q^{j}\right)+q^{d}<q^{d^{2}}=e^{O\left(\ln ^{2} n\right)} .
\end{gathered}
$$

Теорема 6. Зафиксируем $\varepsilon \in(0,1 / 2)$. При $d \rightarrow \infty$ имеем асимптотики:

(a) если $\lambda=m / n \in[\varepsilon, 1-\varepsilon]$, то

$$
n_{m}(A G L(d, q)) \sim \frac{\left(\begin{array}{c}
n \\
m
\end{array}\right)}{|A G L(d, q)|} ;
$$

(b) $е с л и \eta=m /(n+m) \in[\varepsilon, 1-\varepsilon], m o$

$$
n_{(m)}(A G L(d, q)) \sim \frac{\left(\begin{array}{c}
n+m-1 \\
m
\end{array}\right)}{|A G L(d, q)|} .
$$


Более точно, отклонение от 1 среднего значения стабилизатора действия группь $A G L(d, q)$ на соответствующем множестве удовлетворяет оценкам:

в случае $(a)$

$$
\left\langle\operatorname{Stab}_{m}\right\rangle-1 \leqslant \exp \left\{n\left(1-q^{-1}\right) \ln \xi_{1}(\lambda)+\ln ^{2} n\right\}
$$

в случае $(b)$

$$
\left\langle\operatorname{Stab}_{(m)}\right\rangle-1 \leqslant \exp \left\{n\left(1-q^{-1}\right) \ln \xi_{2}(\eta)+\ln ^{2} n\right\},
$$

где $\xi_{1}(\lambda)$ и $\xi_{2}(\eta)$ - числа между 0 и 1, определенные формулой (14), а C - некоторая константа.

Доказательство. Минимальная степень группы $G$ есть $\mu=q^{d}-q^{d-1}=n\left(1-q^{-1}\right)$. (cp. [20], Exercise 3.3.3). Действительно, неподвижные точки аффинного отображения $v \mapsto A v+t$ образуют аффинное подпространство, параллельное линейному подпространству $\operatorname{Ker}(A-I)$; его мощность не больше $q^{d-1}$ при $A \neq I$. Если же $A=I$, но $t \neq 0$, то неподвижных точек нет.

Остается применить теорему 4.

Замечание. (а) При переходе от группы $G$ к ее подгруппе, действующей на том же множестве, размеры стабилизаторов не увеличиваются. В частности, оценки (28) u (27) справедливы для любой подгруппы афбинной группь, в частности, для любой линейной группь, действующей на пространстве $V=\left(\mathbb{F}_{q}\right)^{d}$. Назовем теоремой $6^{\prime}$ копию теоремы 6 с группой $G=G L(d, q)$ вместо $G=A G L(d, q)$.

(b) Напротив, при ограничении действия группы $G$ на $(G$-инвариантное) подмножество $\Omega^{\prime} \subset \Omega$ нет оснований для того, чтобы имеющиеся для $\Omega_{m}$ и $\Omega_{(m)}$ оценки среднего размера стабилизаторов перенести на $\Omega_{m}^{\prime}$ и $\Omega_{(m)}^{\prime}$.

Пример: теорема 6 не является логическим следствием (во всяком случае, простым) теоремы $6^{\prime}$. Подробнее: зафиксировав вложение $V=\left(\mathbb{F}_{q}\right)^{d}$ в $\Omega=\left(\mathbb{F}_{q}\right)^{d+1}$, реализуем полную аффинную группу $A G L(d, q)$ как подгруппу группы $G L(d+1, q)$, сохраняющую подпространство $V$. Согласно пункту (а) замечания, теорема $6^{\prime}$ в размерности $d+1$ влечет те же оценки применительно к действию $A G L(d, q)$ на $\Omega_{m}$ и $\Omega_{(m)}$. Однако было бы логической ошибкой перенести эти оценки на естественное действие $A G L(d, q)$ на $m$-точечных конфигурациях в пространстве $V$, поскольку потребовалось бы ограничить «базовое» действие с $\left(\mathbb{F}_{q}\right)^{d+1}$ на $\left(\mathbb{F}_{q}\right)^{d}$.

5.2. Перечисление графов и гиперграфов Зафиксируем натуральное число $\ell \geqslant 2$. Пусть $X$ - конечное множество, $|X|=n$; будем считать $n$ большим параметром. Положим $\Omega=X_{\ell}$ (множество $\ell$-элементных подможеств в $X$ ). Элементы множества $\Omega_{m}$ называются помеченными однородными $\ell$-гиперграфами на $X$ c гиперребрами [18]. При $\ell=2$ это помеченные графы с $m$ ребрами.

На $\Omega$ естественным образом действует группа $\operatorname{Sym}(X)$. Ее гомоморфный образ в полной группе перестановок $\operatorname{Sym}(\Omega)$ будем обозначать $S_{n}^{\ell}$. Как абстрактные группы, $\operatorname{Sym}(\Omega)$ и $S_{n}^{\ell}$ (при $\left.\ell \leqslant n-1\right)$ изоморфны полной симметрической группе на $n$ индексах, и мы будем говорить о действии элемента (перестановки) $\sigma$ на $X$ или $\Omega$, имея в виду естественный изоморфизм $\operatorname{Sym}(X) \rightarrow S_{n}^{\ell} \subset \operatorname{Sym}(\Omega)$. Что касается естественного действия $S_{n}^{\ell}$ на $\operatorname{Sym}\left(\Omega_{m}\right)$, то специальное обозначение для соответствующей группы перестановок - гомоморфного образа $S_{n}^{\ell}$ в $\operatorname{Sym}\left(\Omega_{m}\right)$ - нам не потребуется. Однако говорить о цикловой структуре элемента $\sigma$ можно лишь зная, 
о действии на каком множестве идет речь. Особенно внимательно за этим надо следить в доказательстве теоремы 8 ниже.

Рассмотрим задачу об асимптотическом перечислении непомеченных однородных гиперграфов с $n$ вершинами и $m$ гиперребрами, т.е. $S_{n}^{\ell}$-орбит на $\Omega_{m}$.

Дальнейшие результаты основаны на асимптотической свободе действия, но случаи $\ell \geqslant 3$ и $\ell=2$ требуют разных подходов: в первом достаточно оценить минимальную степень $S_{n}^{\ell}$ (действующей на $\Omega$ ) и воспользоваться теоремой 4 , в то время как во втором мы прибегнем к теореме 5 . Все дело в соотношении величин минимальной степени и логарифма порядка группы. При всех $\ell \geqslant 2$ минимальная степень имеет порядок $n^{\ell-1}, \operatorname{a} \log |G| \sim n \log n$, так что при $\ell \geqslant 3$ имеем $\mu \gg \log |G|$, а при $\ell=2$ соотношение обратное.

Теорема 7. Пусть фиксированы $\ell \geqslant 3 u \varepsilon \in(0,1 / 2)$. Число $\left|\Omega_{m} / S_{n}^{\ell}\right|$ непомеченных однородных $\ell$-гиперграфов с $п$ вершинами и $m$ гиперребрами имеет асимптотику npu $n \rightarrow \infty$

$$
\left|\Omega_{m} / S_{n}^{\ell}\right|=\frac{\left|\Omega_{m}\right|}{n !}\left(1+\exp \left\{-\frac{2 n^{l-1}}{(l-1) !}(1-o(1))\right\}\right)
$$

если $\varepsilon \leqslant m /|\Omega| \leqslant 1-\varepsilon$. Здесъ

$$
|\Omega|=\left(\begin{array}{c}
n \\
\ell
\end{array}\right) \quad \text { u } \quad\left|\Omega_{m}\right|=\left(\begin{array}{c}
|\Omega| \\
m
\end{array}\right) .
$$

Доказательство. Поскольку $\log \left|S_{n}^{\ell}\right|=\log n !=O(n \log n)$, доказываемое утверждение следует из теоремы 4 и оценки минимальной степени группы $S_{n}^{\ell}$ :

$$
\mu \geqslant \frac{2 n^{l-1}}{(l-1) !}(1-o(1)) .
$$

Докажем ее. Пусть $\sigma \in \operatorname{Sym}(X),|X|=n$, и $j_{k}=j_{k}(\sigma)$ - число циклов длины $k$, ср. $\S 2$, п. 2. Нам нужно оценить сверху число неподвижных точек действия $\sigma$ на $X_{\ell}$. (Выведенная в $\S 2$ оценка $(9)$ в данной ситуации неэффективна.) Искомое число $\alpha_{m}(\sigma)$ - коэффициент при $t^{\ell}$ в произведении $\prod_{k=1}^{\ell} p_{k}(t)$, где $p_{k}(t)$ - часть биномиального разложения $\left(1+t^{k}\right)^{j_{k}}$, включающая только члены степени не выше $\ell$. Запишем $p_{k}(t)=\sum_{i=0}^{\ell} c_{k, i} t^{i}$. Ясно, что $c_{k, i}=0$, если $k$ не делит $i$, а

$$
c_{k, k q}=\left(\begin{array}{c}
j_{k} \\
q
\end{array}\right) \leqslant \frac{n^{q}}{q !} .
$$

Имеем

$$
\alpha_{\ell}(\sigma)=\sum c_{1, i_{1}} c_{2, i_{2}} \ldots c_{\ell, i_{\ell}}
$$

суммирование ведется по таким индексным наборам $\left(i_{1}, i_{2}, \ldots, i_{\ell}\right)$, для которых $i_{k}=$ $k q_{k}$ и $i_{1}+i_{2}+\ldots+i_{\ell}=\ell$. Число всех таких индексных наборов не превосходит $\ell(\ell+1)=O(1)$. Для произведения коэффициентов имеем оценку

$$
c_{1, i_{1}} c_{2, i_{2}} \ldots c_{\ell, i_{\ell}} \leqslant \frac{n^{q_{1}+q_{2}+\ldots+q_{\ell}}}{q_{1} ! \ldots q_{\ell} !} .
$$

Посмотрим внимательно на показатель степени $Q=q_{1}+q_{2}+\ldots+q_{\ell}$ в правой части. Заметим, что $Q=\ell-q_{2}-2 q_{3}-\ldots-(\ell-1) q_{\ell}$. Возможны случаи:

1) $Q=\ell$; так будет, если $q_{1}=\ell, q_{2}=q_{3}=\ldots=0$. 
2) $Q=\ell-1$; так будет, если $q_{1}=\ell-2, q_{2}=1, q_{3}=\ldots=0$.

3) $Q \leqslant \ell-2$ для прочих индексных наборов.

Следовательно,

$$
\alpha_{\ell}(\sigma)=c_{1, \ell}+c_{1, \ell-1} c_{2,2}+O\left(n^{\ell-2}\right)=\left(\begin{array}{c}
j_{1} \\
\ell
\end{array}\right)+\left(\begin{array}{c}
j_{1} \\
\ell-2
\end{array}\right)\left(\begin{array}{c}
j_{2} \\
1
\end{array}\right)+O\left(n^{\ell-2}\right) .
$$

При $\sigma \neq e$ имеем $j_{1} \leqslant n-2$ и, поскольку $j_{1}+2 j_{2} \leqslant n$, легко видеть, что вклад второго слагамого в правой части есть $O\left(n^{\ell-2}\right)$. Получаем нижнюю оценку степени перестановки $\sigma \in S_{n}^{2}$

$$
\beta_{\ell}(\sigma) \geqslant|\Omega|-\left(\begin{array}{c}
n-2 \\
\ell
\end{array}\right)+O\left(n^{\ell-2}\right)=\left(\begin{array}{c}
n \\
\ell
\end{array}\right)-\left(\begin{array}{c}
n-2 \\
\ell
\end{array}\right)+O\left(n^{\ell-2}\right)=\frac{2 n^{\ell-1}}{(\ell-1) !}+O\left(n^{\ell-2}\right),
$$

что и требовалось.

Классический случай $\ell=2$ (перечисление графов) труднее в указанном ранее смысле: оценки, основанной только на минимальной степени, здесь недостаточно. Вступает в игру технический прием «изоляции плохого малого множества». (Следующая теорема - простая версия известных более сильных результатов [19, 3], в которых допускается определенная зависимость $\varepsilon$ от $n$.)

Теорема 8. Пусть $\ell=2$ и фиксировано $\varepsilon \in(0,1 / 2)$. Число $g_{n, m}=\left|\Omega_{m} / S_{n}^{2}\right|$ непомеченных графов с $n$ вершинами и $m$ ребрами имеет асимптотику при $n \rightarrow \infty$

$$
g_{n, m}=\frac{\left|\Omega_{m}\right|}{n !}\left(1+\xi_{1}(\lambda)^{2 n} n^{O(1)}\right)
$$

в предположении $\varepsilon \leqslant \lambda \leqslant 1-\varepsilon$. Здесъ

$$
\lambda=\frac{m}{|\Omega|}, \quad|\Omega|=\left(\begin{array}{c}
n \\
2
\end{array}\right), \quad\left|\Omega_{m}\right|=\left(\begin{array}{c}
|\Omega| \\
m
\end{array}\right),
$$

$u \xi_{1}(\lambda)$ определено в (14).

Доказательство. Степень группы $S_{n}^{(2)}$ равна $\mu=2 n-4$. (Ср. доказательство теоремы 7 или [20], Exercise 5.3.4.)

Пусть $B=\{\sigma \in \operatorname{Sym}(X) \mid \alpha(\sigma) \geqslant n-r\}$, где параметр $r=o(n)$ будет выбран позже. Прежде всего надо решить две задачи:

А. Оценить сверху $|B|$.

В. Рассматривая $B$ уже как подмножество группы $G_{n}^{2}$, оценить снизу его минимальную степень $\mu^{*}=\mu^{S_{n}^{2} \backslash B}$.

Решение задачи A. Пусть $\Sigma_{r}=\left\{(Y, \sigma) \mid Y \in X_{r}, \operatorname{supp}(\sigma) \subset Y\right\}$. Отображение $\Sigma_{r} \rightarrow B_{r}$, заданное формулой $(Y, \sigma) \mapsto \sigma$, сюръективно. (Перестановка $\sigma \in B_{r}$ имеет носитель размера не больших $r$ и есть образ пары $(Y, \sigma)$, где $Y$ - любое $r$-элементное надмножество носителя $\sigma$.) При каждом $Y_{0} \in X_{r}$ слой $\left\{(Y, \sigma) \in \Sigma_{r} \mid Y=Y_{0}\right\}$ отождествляется с группой $\operatorname{Sym}\left(Y_{0}\right)$, т.е. имеет размер $r$ ! Следовательно,

$$
|B| \leqslant\left(\begin{array}{l}
n \\
r
\end{array}\right) r ! \leqslant n^{r}
$$


Решение задачи $B$. Здесь рассматриваем действие $\sigma \in S y m(X) \backslash B$ параллельно на $X$ и на $\Omega=X_{2}$. Величины $j_{1}(\sigma)=\alpha(\sigma), j_{2}(\sigma), \beta(\sigma)$ относятся к действию на $X$, а величины $\alpha_{2}(\sigma)($ ср. $(8))$ и $\left|X_{2}\right|-\alpha_{2}(\sigma)=: \beta_{2}(\sigma)$ относятся к действию на $X_{2}$.

Неподвижные точки действия $\sigma$ на $\Omega$ бывают двух типов:

1) $\left(\begin{array}{c}\alpha(\sigma) \\ 2\end{array}\right)$ пар $(i, j)$, где $i$ и $j-$ неподвижные точки $\sigma$ на $X$;

2) $j_{2}(\sigma)$ пар $(i, j)$, соответствующих 2-циклам $\sigma$ на $X$.

Итак,

$$
\alpha_{2}(\sigma)=\left(\begin{array}{c}
j_{1}(\sigma) \\
2
\end{array}\right)+j_{2}(\sigma)
$$

Отсюда

$$
\beta_{2}(\sigma)=\left(\begin{array}{l}
n \\
2
\end{array}\right)-\left(\begin{array}{c}
j_{1}(\sigma) \\
2
\end{array}\right)-j_{2}(\sigma)=\frac{\beta(\sigma)(n+\beta(\sigma)-1)}{2}-j_{2}(\sigma) .
$$

Если $\sigma \in \operatorname{Sym}(X) \backslash B$, то $\beta(\sigma)>r$. Всегда $j_{2}(\sigma) \leqslant n / 2$. Значит,

$$
\beta_{2}(\sigma)>\frac{r n}{2}-\frac{n}{2}=\frac{n(r-1)}{2} .
$$

Задача В решена: получена оценка

$$
\mu^{*}=\mu^{S_{n}^{2} \backslash B}>\frac{n(r-1)}{2} .
$$

Зафиксируем $q \in(0,1)$ и возьмем $r=\left\lfloor n^{q}\right\rfloor$. Тогда для некоторой константы $C<\infty$

$$
\mu^{*} \ln \xi_{1}(\lambda)+\ln \left|S_{n}^{2}\right| \leqslant n^{q+1} \ln \xi_{1}(\lambda)+C n \ln n
$$

и

$$
\mu \ln \xi_{1}(\lambda)+\log |B|=2 n \ln \xi_{1}(\lambda)+C \ln n .
$$

После подстановки этих оценок в правую часть неравенства (24) теоремы 5 будет доминировать слагаемое $\exp \left\{2 n \ln \xi_{1}(\lambda)+O(\ln n)\right\}$, поскольку $\xi_{1}(\lambda)<1$. Так приходим к оценке (29).

5.3. Оценки для общих групп перестановок В теории групп перестановок известны универсальные оценки минимальной степени $\mu$ снизу и порядка группы $|G|$ сверху в терминах степени $n=|\Omega|$ (размера пространства действия). Это как раз входная информация для теоремы 4. В пункте 2 этого раздела (с. 117) покажем, что теорема 4 дает содержательное усиление тривиальной верхней оценки в (21) для действий больших групп на множествах больших (мульти)множеств в случае, когда базовая группа перестановок примитивна или 2-транзитивна, а в остальном - почти произвольна. Исключение составляют полная симметрическая и знакопеременная группы. В пункте 1 для полноты картины анализируем качество оценок теоремы 4 в этих исключительных случаях.

1. Рассмотрим полную симметрическую группу $G=\operatorname{Sym}(\Omega)$. (Случай знакопеременной группы $G=\operatorname{Alt}(\Omega)$ полностью аналогичен.) Действие $\operatorname{Sym}(\Omega)$ на $\Omega_{m}$ и $\Omega_{(m)}$ наиболее далеко от свободного. Разумеется, интерес здесь представляет не заключение теоремы 4, а лишь сравнение даваемых ею оценок с истинным числом орбит по порядку величины: это позволит судить о том, насколько грубы предсказания теоремы в максимально неблагоприятной ситуации. 
(а) Действие $\operatorname{Sym}(\Omega)$ на $\Omega_{m}$ транзитивно при всех $m \leqslant n$, следовательно, $\left|\Omega_{m} / G\right|=1$ и

$$
\frac{1}{|G|}\left\langle\operatorname{Stab}_{m}^{G}\right\rangle=\frac{1}{\left|\Omega_{m}\right|} \sim e^{-n h(\lambda)} n^{1 / 2} \cdot \operatorname{const}(\lambda)
$$

(cм. (3)).

Минимальная степень полной симметрической группы, $\mu=2$, не растет с ростом порядка группы. Неравенство (22) дает лишь (неинформативную) оценку

$$
\frac{1}{|G|}\left\langle\operatorname{Stab}_{m}^{G}\right\rangle \leqslant n^{1 / 2} \cdot \operatorname{const}(\lambda)
$$

Мы видим, что оценка (22) завышена, по порядку величины, в

$$
e^{n h(\lambda)} \sim|G|^{h(\lambda) / \ln \ln |G|}
$$

раз. (Согласно формуле Стирлинга $n \sim \ln |G| / \ln \ln |G|$.)

$\mathrm{C}$ помощью теоремы 5 можно сократить указанный разрыв. Так же, как в доказательстве Теоремы 8, выделим «плохое» множество $B \subset G$ с тем, чтобы $\mu^{G \backslash B} \geqslant r$; теперь берем $r=t n$, где $t<1$ - параметр. Имеем согласно (30): $|B| \leqslant n^{t n}$. В правой части неравенства (24) первое слагаемое асимптотически доминирует, однако наличие множителя $\xi_{1}^{\mu^{*}}$, где $\mu^{*}=t n$, в отличие от $\mu$, растет вместе с $n$, приводит к оценке вида

$$
\frac{1}{|G|}\left\langle\operatorname{Stab}_{m}^{G}\right\rangle \leqslant e^{t n \ln \xi_{1}(\lambda)} n^{1 / 2} \cdot \operatorname{const}(\lambda),
$$

которая, заметим, уже информативна, т.е. лучше тривиальной. Эта оценка завышена, по порядку величины, в

$$
e^{n q(t, \lambda)} \sim|G|^{q(t, \lambda) / \ln \ln |G|}
$$

раз, где

$$
q(t, \lambda)=h(\lambda)+t \ln \xi_{1}(\lambda) .
$$

Беря $t$ сколь угодно близким к 1 , можем при $\lambda=1 / 2$ сделать $q(t, \lambda)$ сколь угодно близким к $h(\lambda) / 2$. Добиться большего теорема 5 не позволяет. Резерв улучшения оценок за счет подстановки некоторого $t \neq t_{*}$ в (12) при доказательстве теоремы 1 мы в настоящей работе не обсуждаем.

(b) Действие $\operatorname{Sym}(\Omega)$ на $\Omega_{(m)}$ не будет транзитивным, за исключением тривиальных малоразмерных случаев. Орбиты действия находятся во взаимно-однозначном соответствии с разбиениями

$$
m=1 \cdot u_{1}+2 \cdot u_{2}+\ldots
$$

на не более чем $n$ слагаемых:

$$
n \geqslant u_{1}+u_{2}+\ldots
$$

Мультимножества, составляющие орбиту с параметрами $\left(u_{1}, u_{2} \ldots\right)$, содержат ровно $u_{k}$ элементов кратности $k=1,2, \ldots$

Известно [21], что для большинства разбиений числа $m$ количество слагаемых имеет порядок величины const $m^{1 / 2} \ln m$. В предположении $m /(m+n) \sim \eta=$ const, принятом в теоремах 4 и 5, ограничение (32) на число слагаемых намного выше 
этой пороговой величины, поэтому интересующее нас число орбит имеет такую же асимптотику, как число разбиений $m$,

$$
\left|\Omega_{(m)} / G\right| \sim p(m) \sim C m^{-1} e^{\pi \sqrt{2 / 3 m}} .
$$

Ввиду того, что рост здесь субэкспоненциальный, асимптотика логарифма среднего размера стабилизатора для $\Omega_{(m)}$, как и для $\Omega_{m}$, определяется формулой Стирлинга, см. (4):

$$
\ln \frac{\left\langle\operatorname{Stab}_{(m)}^{G}\right\rangle}{|G|}=\ln \frac{\left|\Omega_{(m)} / G\right|}{\left|\Omega_{(m)}\right|} \sim-(n+m) h(\eta) .
$$

Выводы о степени завышенности оценок в теоремах 4 и 5 здесь в целом такие же (в логарифмической шкале), как в пункте (а). Некоторый интерес представляет вычисление наибольшего выигрыша, достигаемого за счет применения теоремы 5 . Рассуждая так же, как в п. (а), приходим к задаче сравнения величин, стоящих в показателях экспонент: $(m+n) h(\eta)$ и

$$
(m+n) h(\eta)+n t \ln \xi_{2}(\eta) .
$$

Имеем - см. (14):

$$
\frac{(m+n) h(\eta)+n t \ln \xi_{2}(\eta)}{(m+n) h(\eta)}=1+t \frac{1-\eta}{2 h(\eta)} \ln \left(\frac{1-\eta}{1+\eta}\right) .
$$

Нижняя грань этого выражения (при $0<t<1,0<\eta<1$ ) равна $1 / 2$ и соответствует $t \rightarrow 1, \eta \rightarrow 1$. Таким образом, как и в пункте (a), теорема 5 по сравнению с теоремой 4 позволяет сократить разрыв между истинной и утверждаемой оценкой менее чем вдвое в логарифмической шкале, причем наибольший (близкий к двукратному) выигрыш получается при $\eta \rightarrow 1$, т.е. при $m \gg n$.

2. В случае примитивных групп, отличных от полной симметрической и знакопеременной, теорема 4 ведет к содержательные следствиям.

Группа перестановок $G$ на множестве $\Omega$ примитивна, если ни при каком $k \geqslant 2$ не существует орбиты действия $G$ на $\Omega_{k}$, состоящей из попарно непересекающихся подмножеств. (Обратно, если такая орбита существует, то ее члены называются блоками импримитивности.) Очевидно, что примитивная группа транзитивна. Легко видеть также, что 2-транзитивное действие $G$ (такое, что индуцированное действие на $\Omega_{2}$ транзитивно) всегда примитивно [17; Theorem 9.6], но обратная импликация неверна. Примитивная, но не 2-транзитивная группа называется унипримитивной. Таким образом, любая транзитивная группа либо 2-транзитивна, либо унипримитивна.

В этом пункте опираемся на результаты Бабаи (усиление теоремы Жордана 1871 г.) [22], [20; Theorem 5.3A], [23], [24; p. 2054], Бохерта [25], [26], [17; § 15] и Пибера [27], [20; Theorem 5.6A]. Как и раньше, $\mu$ обозначает минимальную степень $G$.

Теорема Бабаи. Для унипримитивной группь $G$ верны неравенства

$$
\mu>\sqrt{n} / 2
$$

$$
|G|<\exp \left(4 \sqrt{n}(\ln n)^{2}\right)
$$


Комбинация теорем Бохерта и Пибера. Для 2-транзитивной группъ $G \not \supset$ Alt $(\Omega)$ верны неравенства

$$
\mu>\frac{n}{3}-\frac{2}{3} \sqrt{n}
$$

$u$

$$
|G|<\exp \left(72(\ln n)^{3}\right)
$$

Подставляя неравенства Бабаи, Бохерта и Пибера в теорему 4, получим усиление тривиальной верхней оценки среднего размера стабилизатора индуцированного действия $G$ на множествах $\Omega_{m}$ и $\Omega_{(m)}$. Усиление охватывает все достаточно большие транзитивные группы, за исключением Sym и Alt.

Теорема 9. Для любого $\varepsilon \in(0,1 / 2)$ при всех достаточно больших $n>n_{0}(\varepsilon)$ верны следующие утверждения, в которых $n=|\Omega| u \delta \rightarrow 0$ при $n \rightarrow \infty$. Кроме того, полагаем $\xi=\xi_{1}(\lambda)$ для действия на подмножествах и $\xi=\xi_{2}(\eta)$ для действия на мультимножествах; функиии $\xi_{1}(\lambda)$ и $\xi_{2}(\eta)$ определены в (14).

(а) Для естественного действия унипримитивной группы $G$ на подмножествах множества $\Omega$ размера $m \in(n \varepsilon, n(1-\varepsilon))$ и на мультимножествах размера $m \in$ $(n \varepsilon /(1-\varepsilon), n(1-\varepsilon) / \varepsilon)$ существует хотя бы одна орбита $\mathcal{O}$ размера

$$
|\mathcal{O}| \geqslant|G|^{A /(\ln \ln |G|)^{2}}, \quad A=\left(\frac{1}{8}-\delta\right)|\ln \xi| .
$$

(b) Пусть дана 2-транзитивная группа $G$ перестановок множества $\Omega$, отличная от $\operatorname{Sym}(\Omega)$ и Alt $(\Omega)$. Тогда среди всех орбит естественного действия группь $G$ на подмножествах $\Omega$ размера $m \in(n \varepsilon, n(1-\varepsilon))$ и на мультимножествах размеpa $m \in(n \varepsilon /(1-\varepsilon), n(1-\varepsilon) / \varepsilon)$ доля регулярных орбит стремится $\kappa 1$ при $n \rightarrow \infty$. Более точно, отклонение от 1 среднего размера стабилизатора в этих случалх $\left(\langle\operatorname{Stab}\rangle=\left\langle\operatorname{Stab}_{m}^{G}\right\rangle\right.$ или $\left.\left\langle\operatorname{Stab}_{(m)}^{G}\right\rangle\right)$ допускает оченку сверху:

$$
\langle\mathrm{Stab}\rangle-1 \leqslant \xi^{n / 3-(2 / 3+\delta) \sqrt{n}}
$$

Доказательство. Пусть $S$ обозначает $\Omega_{m}$ или $\Omega_{(m)}$, $\langle\operatorname{Stab}\rangle=\left\langle\operatorname{Stab}_{m}^{G}\right\rangle$ или

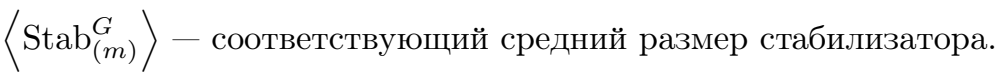

(а) Для унипримитивной группы неравенство (33) и теорема 4 дают

$$
\frac{\langle\text { Stab }\rangle}{|G|} \leqslant \text { const } \cdot \sqrt{n} \cdot \xi^{\sqrt{n} / 2}=\exp \left(\frac{\ln \xi}{2} \sqrt{n}(1+o(1))\right) .
$$

Очевидно неравенство

$$
|S| \leqslant|S / G| \cdot \max _{\mathcal{O} \in S / G}|\mathcal{O}|
$$

Следовательно, существует хотя бы одна орбита $\mathcal{O}$, для которой

$$
|\mathcal{O}| \geqslant \frac{|S|}{|S / G|}=\frac{|G|}{\langle\text { Stab }\rangle} \geqslant \exp \left(\frac{|\ln \xi|}{2} \sqrt{n}(1-o(1))\right) .
$$


Неравенство (34) влечет, как легко видеть,

$$
\sqrt{n} \geqslant \frac{\ln |G|}{4(\ln \ln |G|)^{2}}(1-o(1)) .
$$

Комбинируя два последних неравенства, получаем (37).

(b) Для 2-транзитивной группы $G \not \supset A l t(\Omega)$, используя $(35)$, имеем

$$
\frac{\left\langle\operatorname{Stab}_{m}^{G}\right\rangle-1}{|G|} \leqslant \xi^{n / 3-2 / 3 \sqrt{n}+O(\ln n)},
$$

а согласно $(36),|G|=\exp (o(\sqrt{n}))$. Так получается оценка $(38)$.

Замечание 1. Для широких классов групп с описанными исключениями существуют более точные оценки по сравнению с использованными в последнем доказательстве - см., например, $[28,29]$. Интересно также было бы исследовать вопрос об улучшении оценок в направлении, подсказываемом теоремой 5. Помимо ранее цитированных работ, содержащих результаты и ссылки, которые могут при этом оказаться полезными, укажем на обзор на русском языке [30].

Замечание 2. Действие полной аффинной группы, рассмотренное в разд. 5.1, 2-транзитивно, поэтому вариант теоремы 6 с несколько худшими остаточными членами является следствием теоремы 9b. Напротив, действие группы $G=\operatorname{Sym}(V)$ перенумерации вершин графа на $\Omega=V_{2}$ унипримитивно, и заключение теоремы 8 намного сильнее, чем общей теоремы 9а. Это не удивительно: доказательство теоремы 8 опирается не только на оценку минимальной степени группы, но и на дополнительную информацию, позволяющую отделить множество $B$.

\section{Список литературы}

1. Харари Ф., Палмер Э., Перечисление графов, Мир, Москва, 1977.

2. Ford G. W., Uhlenbeck G. E., "Combinatorial problems in the theory of graphs IV", Proc. Nat. Acad. Sci. USA, 43 (1957), 163-167.

3. Wright E. M., "Graphs on unlabelled nodes with a given number of edges", Acta Math., 168 (1971), 1-9.

4. Hoffman F., Welch L., "Totally variant sets in finite groups and vector spaces", Canad. J. Math., 20, 701-710.

5. Strazdins I., "Universal affine classification of Boolean functions", Acta Applic. Math., 46 (1997), 147-167.

6. Wild M., "The asymptotic number of inequivalent binary codes and nonisomorphic binary matroids", Finite Fields and Their Appl., 6 (2000), 192-202.

7. Wild M., "The asymptotic number of binary codes and binary matroids", SIAM J. Discrete Math., 19:3 (2005), 691-699.

8. Xiang-Dong Hou, "On the asymptotic number of non-equivalent binary linear codes", Finite Fields and Their Appl., 13 (2007), 318-326.

9. Xiang-Dong Hou, "On the asymptotic number of inequivalent binary self-dual codes", J. Comb. Theory, Ser. A, 114 (2007), 522-544.

10. Xiang-Dong Hou, "Asymptotic numbers of non-equivalent codes in three notions of equivalence", Linear and Multilinear Algebra, 57:2 (2009), 111-122.

11. Lax R. F., "On the character of $S_{n}$ acting on subspaces of $\mathbb{F}_{n}$ ", Finite Fields and Their Appl., 10 (2004), 315-322. 
12. Cameron P. J., "Permutation groups on unordered sets", Higher Combinatorics (Proc. NATO Adv. Study Inst. Series), 31, ed. M. Aigner, D. Reidel Publ. Co., Amsterdam, 1978, 217-239.

13. Cameron P. J., Permutation groups, Cambridge Univ. Press, Cambridge, 1999.

14. Siemons J., "483-487", Arch. Math., 43 (1984).

15. Livingstone D., Wagner A., "393-403", Math. Zeitschr., 90 (1965).

16. Kochetov M., Parsons N., Sadov S., "Counting fine gradings on matrix algebras and on classical simple Lie algebras", Int. J. of Algebra and Computations, 23:7 (2013), 1755-1781.

17. Wielandt H., Finite permutation groups, Academic Press, New York-London, 1964.

18. Duchet P., Hypergraphs, Handbook of Combinatorics, eds. Graham R., Grötschel M., Lovász L., Elsevier Science, Amsterdam-Lausanne-New York-Oxford-Shannon-Tokyo, 1995, 381-432.

19. Коршунов А.Д., "О мощности некоторых классов графов”, Доклады АН CCCP, 193:6 (1970), 1230-1233.

20. Dixon J. D., Mortimer B., Permutation groups, New York, 1996.

21. Erdös P., Lehner J., "The distribution of the number of summands in the partitions of a positive integer", Duke Math. J., 8:2 (1941), 335-345.

22. Babai L., "On the order of uniprimitive permutation groups", Ann. Math., 113:3 (1981), $553-568$.

23. Земляченко В.Н., Корнеенко Н.М., Тышкевич Р.И., "Проблема изоморфизма графов", Теория сложности вычислений. I, Зап. научн. сем. ЛОМИ, 118, 1982, 83-158.

24. Lovász L., Pyber L., Welsh D. J. A., Ziegler G. M., "Combinatorics in Pure Mathematics", Handbook of Combinatorics, eds. Graham R., Grötschel M., Lovász L., Elsevier Science, Amsterdam-Lausanne-New York-Oxford-Shannon-Tokyo, 1995, 2039-2082.

25. Bochert A., "Ueber die Classe der Transitiven Substitutionengruppen II", Math. Ann., 49 (1897), 133-144.

26. Herzog M., Praeger C. E., "Minimal degree of primitive permutation groups. In: Combinatorial Mathematics IV, Adelaide 1975", Lecture Notes in Math., 560, 116-122.

27. Pyber L., "On the orders of doubly transitive permutation groups, elementary estimates.", J. Comb. Theory, Ser. A, 62 (1993), 361-366.

28. Liebeck M. W., Shalev A., "Bases of primitive permutation groups", Groups, Combinatorics and Geometry: Durham, 2001, eds. Ivanov A. A., Liebeck M. W., Saxl J., World Scientific, New Jersey-London-Singapore-Hong Kong, 2003, 147-154.

29. Maróti A., "On the orders of primitive groups", J. Algebra, 258:2 (2002), 631-640.

30. Глухов М. М., Зубов А. Ю., "О длинах симметрических и знакопеременных групп подстановок в различных системах образующих (обзор)", Мат. вопр. кибернетики, 8, Наука, Москва, 2000, 3-30. 